\title{
ON KODAIRA VANISHING FOR SINGULAR VARIETIES
}

\author{
DONU ARAPURA AND DAVID B. JAFFE
}

(Communicated by Louis J. Ratliff, Jr.)

\begin{abstract}
If $X$ is a complex projective variety with an ample line bundle $\mathscr{L}$, we show that $H^{i}\left(X, \mathscr{L}^{-1}\right)=0$ for any $i<\operatorname{codim}[\operatorname{Sing}(X)]$, provided that $X$ satisfies Serre's condition $S_{i+1}$. We also give examples to show that these results are sharp. Finally, we prove a vanishing theorem (for $H^{1}$ ) for seminormal varieties
\end{abstract}

\section{INTRODUCTION}

Consider a complex projective variety $X$, together with an ample line bundle $\mathscr{L}$. Kodaira [4] has proved that if $X$ is nonsingular, then $H^{i}\left(X, \mathscr{L}^{-1}\right)=0$ for all $i<\operatorname{dim}(X)$. To what extent is this true when $X$ is singular? If one is only concerned with the depth and the dimension of the singularities of $X$, then there is a simple answer: $H^{i}$ vanishes provided that $i<\operatorname{codim}[\operatorname{Sing}(X)]$ and $X$ satisfies property $S_{i+1}$ of Serre. If $X$ is Cohen-Macaulay, this was known: see $[8, \S 7.80]$. We reduce to this case easily. Our result generalizes a theorem of Mumford [7], who proved that if $X$ is normal (of dimension at least two), then $H^{1}$ vanishes.

The above vanishing criterion cannot be improved in any naive way. Grothendieck knew that the depth condition was essential [3, XII 1.3]. The regularity condition is also essential: for any integers $0<i<n$, there exists a projective Cohen-Macaulay variety $X$ of dimension $n$, such that $\operatorname{codim}[\operatorname{Sing}(X)]$ $=i$, together with an ample line bundle $\mathscr{L}$ such that $H^{i}\left(X, \mathscr{L}^{-1}\right) \neq 0$. If $i>1$, these examples are trivial variants of an example of Sommese [9]. For $i=1$, a different construction is needed.

One may search for more delicate local criteria for vanishing. For instance, a consequence of [2] is that $H^{i}$ vanishes if $X$ has rational singularities: see [8]. By definition, if $X$ is rational, it is normal, so this criterion is not applicable to singularities which live in codimension one. We prove a theorem which applies

Received by the editors May 2, 1988 and, in revised form, July 18, 1988.

1980 Mathematics Subject Classification (1985 Revision). Primary 32L20, 14 F99.

Key words and phrases. Vanishing theorem, seminormal.

The first author was partially supported by the National Science Foundation 
to exactly this situation: if $X$ is seminormal, $S_{2}$, and has dimension at least two, then $H^{1}\left(X, \mathscr{L}^{-1}\right)$ vanishes.

In this paper, we shall assume that all varieties are defined over $\mathbf{C}$.

\section{VANISHING BASED ON REGULARITY AND DEPTH}

We prove:

Proposition 1.1. Let $X$ be a projective variety, and let $\mathscr{L}$ be an ample line bundle on $X$. Fix $k \geq 1$. Assume that $k<\operatorname{codim}[\operatorname{Sing}(X)]$ and that $X$ is $S_{k+1}$. Then $H^{k}\left(X, \mathscr{L}^{-1}\right)=0$. (If $X$ is smooth, we define $\operatorname{codim}[\operatorname{Sing}(X)]=$ $\operatorname{dim}(X)$.)

Proof. The statement was known if $X$ is Cohen-Macaulay. (See $[8, \S 7.80]$.) Let $n=\operatorname{dim}(X)$. Let $H \subset X$ be a sufficiently general and sufficiently ample hyperplane. We have an exact sequence

$$
\left.0 \rightarrow \mathscr{L}^{-1}(-H) \rightarrow \mathscr{L}^{-1} \rightarrow \mathscr{L}^{-1}\right|_{H} \rightarrow 0,
$$

and taking cohomology we obtain

$$
H^{k}\left(\mathscr{L}^{-1}(-H)\right) \rightarrow H^{k}\left(\mathscr{L}^{-1}\right) \rightarrow H^{k}\left(\left.\mathscr{L}^{-1}\right|_{H}\right)
$$

We may assume that $X$ is not Cohen-Macaulay and hence that $k+1<$ $\operatorname{dim}(X)$. This, together with the appropriate Bertini theorems imply that $k<$ $\operatorname{codim}[\operatorname{Sing}(H)]$ and that $H$ is $S_{k+1}$. Since $X$ is $S_{k+1}$, a result of Grothendieck [3, XII 1.3], implies that $H^{k}\left(\mathscr{L}^{-1}(-H)\right)=0$ so long as $H$ is sufficiently ample. By induction on the dimension $H^{k}\left(\left.\mathscr{L}^{-1}\right|_{H}\right)$ is zero, hence the result.

Remark 1.2. Let $\operatorname{Irr}(X)$ denote the irrational locus of $X$, given by

$$
\operatorname{Irr}(X)=\bigcup_{i>0} \operatorname{Supp}\left(R^{i} \pi_{*} \mathscr{O}_{\widetilde{X}}\right),
$$

where $\pi: \tilde{X} \rightarrow X$ is a resolution of singularities. In the proposition, one can weaken the hypothesis on the singular locus to the condition that $k<$ $\operatorname{codim}[\operatorname{Irr}(X)]$. The same proof works, allowing one to reduce to the case where $X$ is Cohen-Macaulay, which is well known. One needs to known the following Bertini-type lemma: if $H \subset X$ is a general hyperplane, then $\operatorname{Irr}(X \cap H) \subset \operatorname{Irr}(X) \cap H$. The proof of this lemma is left to the reader.

\section{CountereXAmples}

The result of this section is: Fix integers $0<k<n$. Then there exists a projective Cohen-Macaulay variety $X$ of dimension $n$, with $\operatorname{codim}[\operatorname{Sing}(X)]=$ $k$, and an ample line bundle $\mathscr{L}$ on $X$ such that $H^{k}\left(X, \mathscr{L}^{-1}\right) \neq 0$.

Actually, except for the case $k=1$, this is obtained by a trivial variant of a known construction $[9,0.2 .4]$. The author has kindly informed us of a critical typographical error, so we summarize the construction for the convenience of the reader. 
We first assume that $k>1$. (The case $(n=3, k=2)$ occurs in [9].) Let

$$
P=\mathbf{P}\left(\mathscr{O}_{\mathbf{P}^{n-k}} \oplus\left[\mathscr{O}_{\mathbf{P}^{n-k}}(1)\right]^{\oplus(k+1)}\right) .
$$

Let $\mathscr{T}$ denote the tautological bundle on $P$. Let $X$ be a general member of the linear system associated to $\left[\mathscr{T} \otimes \mathscr{O}_{\mathbf{p}^{n-k}}(-1)\right]^{\otimes(k+2)}$. Let $\mathscr{L}=\mathscr{T} \otimes \mathscr{O}_{\mathbf{p}^{n-k}}(1)$. Then $H^{k}\left(X, \mathscr{L}^{-1}\right) \neq 0$. The fibers of $X \rightarrow \mathbf{P}^{n-k}$ are cones, each having a unique singular point. Some details may be found in the original source.

Now we deal with the case $k=1$. We describe the subcase $n=2$. The construction is easily modified for higher values of $n$.

Let $Y$ be the ruled surface $\mathbf{P}\left(\mathscr{O}_{\mathbf{P}^{1}} \oplus \mathscr{O}_{\mathbf{P}^{1}}(-2)\right)$. Let $C_{0}$ and $C_{\infty}$ be the sections of $\pi: Y \rightarrow \mathbf{P}^{1}$ with self-intersection -2 and 2 , respectively. (So $C_{\infty} \sim C_{0}+2 f$.) Let $f$ be a fiber of $\pi$. Let $X$ be the cyclic double cover of $Y$, branched along the divisor $3 C_{0}+C_{\infty}$. (The divisor $3 C_{0}+C_{\infty}$ is uniquely divisible by 2 in $\operatorname{Pic}(Y): 3 C_{0}+C_{\infty} \sim 2\left(2 C_{0}+f\right)$.) Let $\mathscr{L}$ be the line bundle on $X$ which is the pullback to $X$ of $\mathscr{O}_{Y}\left(C_{0}+3 f\right)$. The singularities of $X$ are analytically of the form $\mathbf{C}[[x, y, t]] /\left(x^{2}+y^{3}\right)$. Then $X$ is Cohen-Macaulay (but not normal), $\mathscr{L}$ is ample, and $H^{1}\left(X, \mathscr{L}^{-1}\right) \neq 0$. (One may calculate that

$$
H^{1}\left(X, \mathscr{L}^{-1}\right) \cong H^{1}\left(Y, \mathscr{O}\left(-C_{0}-3 f\right)\right) \oplus H^{1}\left(Y, \mathscr{O}\left(-3 C_{0}-4 f\right)\right)
$$

and the second summand is nonzero.) The details are left to the reader.

For higher values of $n$, replace $\mathbf{P}^{1}$ by $\mathbf{P}^{n-1}$, and replace $\mathscr{L}$ by $\mathscr{O}_{Y}\left(C_{0}+\right.$ $(n+1) f)$.

\section{VANISHING OF $H^{1}$ FOR SEMINORMAL VARIETIES}

The result of this section is:

Theorem 3.1. Let $S$ be a projective seminormal $S_{2}$ variety of dimension at least two. Let $\mathscr{L}$ be an ample line bundle on $S$. Then $H^{1}\left(S, \mathscr{L}^{-1}\right)=0$.

Remark 3.2. It is known [5, 3.9] that seminormal $S_{2}$ varieties have a particularly simple geometry: they are those $S_{2}$ varieties, which (outside a subset of codimension two) look locally like $N \times \mathbf{C}^{r}$, where $N$ is the union of the coordinate axes in $\mathbf{C}^{k}$. (But $k$ can vary.)

We need the following well-known lemma:

Lemma 3.3. Let $X$ be a reduced projective scheme over $\mathbf{C}$ (having no zero dimensional components). Let $\mathscr{L}$ be an ample line bundle on $X$. Then $H^{0}\left(X, \mathscr{L}^{-1}\right)=0$.

Proof (of Theorem 3.1). We first prove the theorem in the case where $S$ is a surface. Let $\pi: \widetilde{S} \rightarrow S$ be the normalization map. let $\mathscr{I}$ be the conductor of $\mathscr{O}_{\widetilde{S}}$ into $\mathscr{O}_{S}$. It is a sheaf of ideals in both $\mathscr{O}_{S}$ and in $\mathscr{O}_{\widetilde{S}}$. Let $\Delta$ be the closed subscheme of $S$ determined by $\mathscr{I}$, and let $\widetilde{\Delta}$ be the closed subscheme of $\widetilde{S}$ determined by $\mathscr{J}$. By $[6,1.5]$ or $[11,1.3] \Delta$ and $\widetilde{\Delta}$ are reduced schemes. (This is the only place where we use the hypothesis that $S$ is seminormal.) 
We shall make various calculations with $\mathscr{O}_{S}$-modules, and we shall write for instance $\mathscr{Q}_{\widetilde{S}}$ instead of $\pi_{*} \mathscr{Q}_{\widetilde{S}}$, to avoid cumbersome notation.

There is an exact sequence of $\mathscr{O}_{S}$-modules

$$
0 \rightarrow \mathscr{O}_{S} \stackrel{p}{\longrightarrow} \mathscr{O}_{\widetilde{S}} \oplus \mathscr{O}_{\Delta} \stackrel{q}{\longrightarrow} \mathscr{O}_{\widetilde{\Delta}} \rightarrow 0
$$

which is obtained from the canonical maps of $\mathscr{O}_{S}$-modules:

$$
\begin{aligned}
& p_{1}: \mathscr{O}_{S} \rightarrow \mathscr{O}_{\widetilde{S}} \\
& p_{2}: \mathscr{O}_{S} \rightarrow \mathscr{O}_{\Delta} \\
& q_{1}: \mathscr{O}_{\widetilde{S}} \rightarrow \mathscr{O}_{\widetilde{\Delta}} \\
& q_{2}: \mathscr{O}_{\Delta} \rightarrow \mathscr{O}_{\widetilde{\Delta}}
\end{aligned}
$$

by $p=p_{1}+p_{2}$ and $q=q_{1}-q_{2}$. It is easy to verify that the sequence is in fact exact. (This exact sequence has been used by Steenbrink [10], proof of theorem 3.)

Tensor the given exact sequence by $\mathscr{L}^{-1}$ and compute the long exact sequence of cohomology on $S$. By $(3.3), H^{0}\left(\mathscr{L}^{-1} \otimes \mathscr{Q}_{\bar{\Delta}}\right)=0$. Hence we have an exact sequence:

$$
0 \rightarrow H^{1}\left(\mathscr{L}^{-1}\right) \rightarrow H^{1}\left(\mathscr{L}^{-1} \otimes \mathscr{O}_{\widetilde{S}}\right) \oplus H^{1}\left(\mathscr{L}^{-1} \otimes \mathscr{O}_{\Delta}\right) \stackrel{\phi}{\longrightarrow} H^{1}\left(\mathscr{L}^{-1} \otimes \mathscr{O}_{\Delta}\right) .
$$

We must show that $\phi$ is injective. Observe that $H^{1}\left(\mathscr{L}^{-1} \otimes \mathscr{Q}_{\widetilde{S}}\right)$ is isomorphic to $H^{1}\left(\tilde{S}, \pi^{*} \mathscr{L}^{-1}\right)$. This is $H^{1}$ of the dual of an ample invertible sheaf on a normal surface, which by a theorem of Mumford [7] is zero. (This also follows from 1.1.)

We have exact sequences:

$$
0 \rightarrow \mathscr{O}_{\Delta} \rightarrow \mathscr{O}_{\Delta_{\text {nor }}} \rightarrow \mathscr{M} \rightarrow 0
$$

and

$$
0 \rightarrow \mathscr{Q}_{\widetilde{\Delta}} \rightarrow \mathscr{Q}_{\widetilde{\Delta}_{\text {nor }}} \rightarrow \widetilde{\mathscr{M}} \rightarrow 0 .
$$

Then $\mathscr{M}$ and $\widetilde{\mathscr{M}}$ have finite support, so $\mathscr{M} \cong \mathscr{M} \otimes \mathscr{L}^{-1}$ and $\widetilde{\mathscr{M}} \cong \widetilde{\mathscr{M}} \otimes \mathscr{L}^{-1}$, noncanonically. We obtain:

$$
\begin{aligned}
& 0 \rightarrow H^{0}(\mathscr{M}) \rightarrow H^{1}\left(\mathscr{L}^{-1} \otimes \mathscr{O}_{\Delta}\right) \rightarrow H^{1}\left(\mathscr{L}^{-1} \otimes \mathscr{O}_{\Delta_{\text {nor }}}\right) \rightarrow 0 \\
& \downarrow \rho_{1}(\mathscr{L}) \quad \downarrow \rho_{2}(\mathscr{L}) \quad \downarrow \rho_{3}(\mathscr{L}) \\
& 0 \rightarrow H^{0}(\widetilde{\mathscr{M}}) \rightarrow H^{1}\left(\mathscr{L}^{-1} \otimes \mathscr{Q}_{\Delta}\right) \rightarrow H^{1}\left(\mathscr{L}^{-1} \otimes \mathscr{Q}_{\widetilde{\Delta}_{\text {nor }}}\right) \rightarrow 0 .
\end{aligned}
$$

We want to prove that $\rho_{2}(\mathscr{L})$ is injective. It suffices to show that $\rho_{3}(\mathscr{L})$ and $\rho_{1}(\mathscr{L})$ are injective. Now $\rho_{3}(\mathscr{L})$ is injective because $\mathscr{O}_{\Delta_{\text {nor }}}$ is a smooth curve (perhaps disconnected) and hence the map

$$
\mathscr{O}_{\Delta_{\text {nor }}} \rightarrow \mathscr{O}_{\widetilde{\Delta}_{\text {nor }}}
$$

of $\mathscr{O}_{\Delta_{\text {nor }}}$-modules is split injective (via trace). Because $S$ is Cohen-Macaulay, the Serre duality and Serre vanishing theorems imply that $H^{1}\left(\mathscr{L}^{-n}\right)=0$ for $n \gg 0$. Hence $\rho_{2}\left(\mathscr{L}^{n}\right)$ is injective for $n \gg 0$. Hence $\rho_{1}\left(\mathscr{L}^{n}\right)$ is injective 
for $n \gg 0$. But $\rho_{1}(\mathscr{L})$ is independent of $\mathscr{L}$, so in fact $\rho_{1}(\mathscr{L})$ is injective. Hence $\rho_{2}(\mathscr{L})=\phi$ is injective. This proves the theorem in the case where $S$ is a surface.

Now we prove the theorem in the general case, by induction on the dimension of $S$. If $\operatorname{dim}(S)=2$ we are done. Otherwise, embed $S$ in some projective space $\mathbf{P}$. Since $S$ is $S_{2}$, we know by the lemma of Enriques-Severi-Zariski that $H^{1}\left(S, \mathscr{L}^{-1}(-n)\right)=0$ for $n \gg 0$. Changing the embedding, we may assume that $H^{1}\left(S, \mathscr{L}^{-1}(-1)\right)=0$. By the Bertini theorem for seminormality $[1,3.9$ or 12], one knows that there exists a hyperplane $H \subset \mathbf{P}$ such that $S \cap H$ is seminormal. Also, we may choose $H$ so that $S \cap H$ is $S_{2}$. Tensor the exact sequence:

$$
0 \rightarrow \mathscr{O}_{S}(-1) \rightarrow \mathscr{O}_{S} \rightarrow \mathscr{O}_{S \cap H} \rightarrow 0
$$

by $\mathscr{L}^{-1}$ and compute $H^{1}$. The theorem follows immediately.

Remark 3.4. If the ground field has positive characteristic, by adding suitable hypotheses one can still make the proof work: let $S$ be a projective seminormal Cohen-Macaulay surface. Assume that the Picard scheme $\operatorname{Pic}\left(S_{\text {nor }}\right)$ is reduced. Let $\mathscr{L}$ be an ample line bundle on $S$. Then $H^{1}\left(S, \mathscr{L}^{-1}\right)=0$.

\section{ACKNOWLEDGMENTS}

Craig Huneke has kindly pointed out some simplifications to the proof of (3.1). Also, the referee has provided a shorter proof of (1.1).

\section{REFERENCES}

1. C. Cumino, S. Greco and M. Manaresi, Bertini theorems for weak normality, Compositio Math. 48 (1983), 351-362.

2. H. Grauert, and O. Riemenschneider, Verschwindungssätze für analytische Kohomologiegruppen auf komplexen Räume, Invent. Math. 11 (1970), 263-292.

3. A. Grothendieck, Cohomologie locale des faisceaux coherents et theorems de Lefschetz locaux et globaux, Séminaire de Géométrie Algébrique (SGA 2), North-Holland, 1968.

4. K. Kodaira, On a differential-geometric method in the theory of analytic stacks, Proc. Nat. Acad. Sci. U.S.A. 39 (1953), 1268-1273.

5. J. V. Leahy and M. A. Vitulli, Weakly normal varieties: The multicross singularity and some vanishing theorems on local cohomology, Nagoya Math. J. 83 (1981), 137-152.

6. __ Seminormal rings and weakly normal varieties, Nagoya Math. J. 82 (1981), 27-56.

7. D. Mumford, Pathologies. III, Amer. J. Math. 89 (1967), 94-104.

8. B. Shiffman and A. J. Sommese, Vanishing theorems on complex manifolds, Birkhäuser, Boston, Massachusetts, 1985.

9. A. J. Sommese, On the adjunction theoretic structure of projective varieties, Lecture Notes in Math., vol. 1194, Springer-Verlag, Berlin and New York, 1986, pp. 175-213. 
10. J. H. M. Steenbrink, Cohomologically insignificant degenerations, Compositio Math. 42 (1981), 315-320.

11. C. Traverso, Seminormality and the Picard group, Ann. Scuola Norm. Sup. Pisa Cl. Sci. (4) 24 (1970), 585-595.

12. M. A. Vitulli, The hyperplane sections of weakly normal varieties, Amer. J. Math. 105 (1983), 1357-1368.

Department of Mathematics, Purdue University, West Lafayette, Indiana 47907 